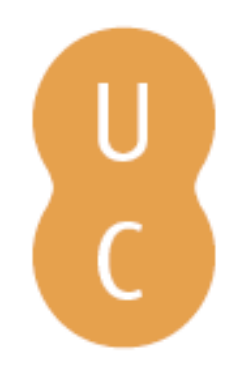

\title{
pompalina
}

\section{Plutarque et l'hermétisme}

Autor(es): $\quad$ Boulogne, Jacques

Publicado por: Imprensa da Universidade de Coimbra; Katholieke Universiteit Leuven

URL

persistente: URI:http://hdl.handle.net/10316.2/32090

DOI: $\quad$ DOI:http://dx.doi.org/10.14195/978-989-26-0462-6_4

Accessed : $\quad$ 26-Apr-2023 14:38:19

A navegação consulta e descarregamento dos títulos inseridos nas Bibliotecas Digitais UC Digitalis, UC Pombalina e UC Impactum, pressupõem a aceitação plena e sem reservas dos Termos e Condições de Uso destas Bibliotecas Digitais, disponíveis em https://digitalis.uc.pt/pt-pt/termos.

Conforme exposto nos referidos Termos e Condições de Uso, o descarregamento de títulos de acesso restrito requer uma licença válida de autorização devendo o utilizador aceder ao(s) documento(s) a partir de um endereço de IP da instituição detentora da supramencionada licença.

Ao utilizador é apenas permitido o descarregamento para uso pessoal, pelo que o emprego do(s) título(s) descarregado(s) para outro fim, designadamente comercial, carece de autorização do respetivo autor ou editor da obra.

Na medida em que todas as obras da UC Digitalis se encontram protegidas pelo Código do Direito de Autor e Direitos Conexos e demais legislação aplicável, toda a cópia, parcial ou total, deste documento, nos casos em que é legalmente admitida, deverá conter ou fazer-se acompanhar por este aviso.

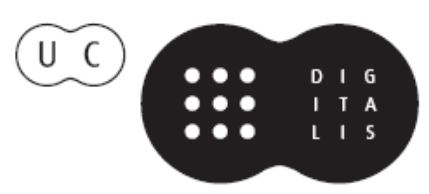




\title{
Puilosophy in Society \\ Virtues and Values in Plutarch
}

\author{
José Ribeiro Ferreira \\ LUC VAN DER STOCKT \\ Maria do Céu Fialho
}

Editors

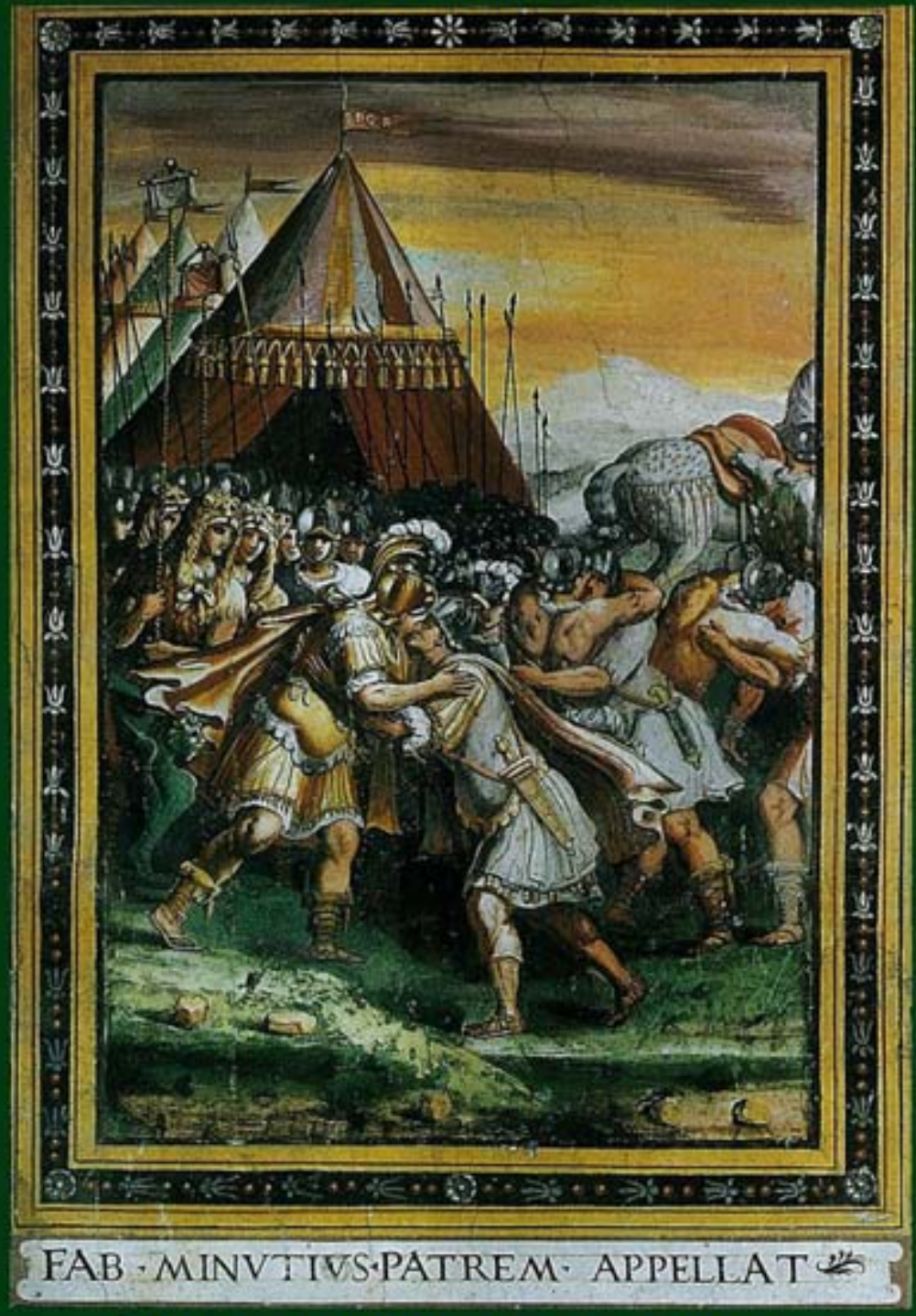

KATHOLIEKE UNIVERSITEIT LEUVEN IMPRENSA DA UNIVERSIDADE DE COIMBRA Leuven-Coimbra, 2008 
(Página deixada propositadamente em branco) 
José Ribeiro Ferreira, Luc van der Stockt \& Maria do Céu Fialho

EDITORS

\section{PhILOSOPHY IN Society}

\section{Virtues and Values in Plutarch}

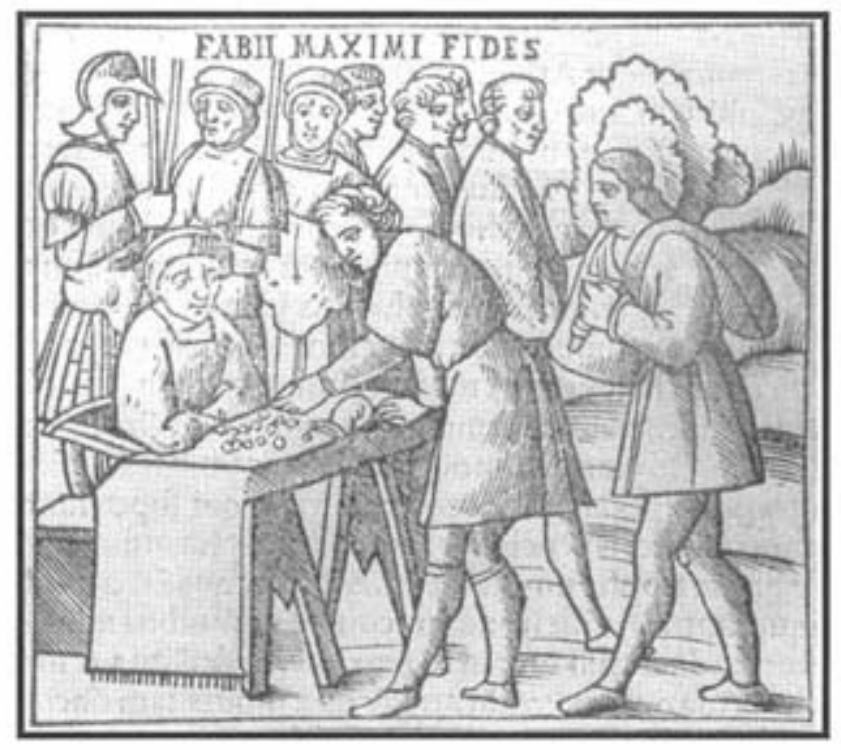

Fabius Maximus' Loyalty

Vitae Plutarchi Cheronei novissime post Jodocum Badium Ascensium longe diligentius repositae maioreque diligentia castigatae, cum copiosiore verioreque indice, nec non cum Aemilii Probi vitis, una cum figuris, suis locis apte dispositis, Venetiis 1516 , fol . 65v

\section{LEUVEN - CoIMBRA \\ 2008}

KATHOLIEKE UNIVERSITEIT LEUVEN

IMPRENSA DA UNIVERSIDADE DE COIMBRA 


\title{
First published 2008
}

\section{(C) Universiteit KATHOlieke Leuven \\ (c) Universidade Coimbra}

\author{
Published by \\ IMPRENSA DA UNIVERSIDADE COIMBRA \\ Imprensa da Universidade de Coimbra \\ Rua da Ilha, $\mathrm{n}^{\circ} 1$ \\ 3000-033 Coimbra (Portugal) \\ Email: imprensauc@ci.uc.pt \\ URL: http://www.uc.pt/imprensa_uc
}

ISBN: 972-989-8074-73-7

Legal Deposit: MA-140-2009

\author{
Printed in Spain by \\ IMAGRAF IMPRESORES, S.A. \\ c/ Nabucco 14 \\ 29006 Málaga \\ Tfno. 952328597
}

\section{Frontispiece:}

Fabius Maximus and Minucius (Francesco da Siena, Grottaferrata, Palazzo Abbaziale). We are grateful to the Archimandrita of the "Monastero Esarchico di Santa Maria di Grottaferrata", P. Emiliano Fabbricatore, for the authorization to reproduce this picture. 


\section{Plutarque et l'hermétisme \\ JACQUES BOULOGNE \\ Université Charles de Gaulle- LiLle 3 \\ HALMA-IPEL - UMR 8164}

Pour justifier les étymologies du nom de divinités égyptiennes fondées sur la langue grecque, Plutarque, dans son traité d'herméneutique, Isis et Osiris $(61,375$ $\mathrm{E}-376 \mathrm{~A}$ ), écrit que beaucoup de termes grecs, du fait de l'émigration hellénique, se retrouvent à l'étranger, notamment en Égypte, sous une forme barbare, et il se

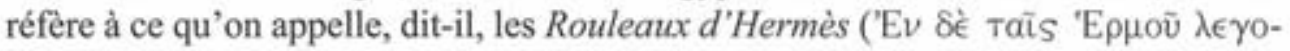

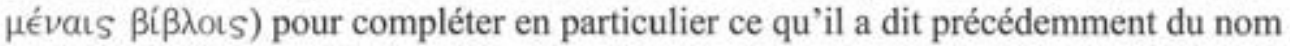
d'Osiris, composé, explique-t-il, de deux adjectifs grecs, hosios et hieros (61, 345

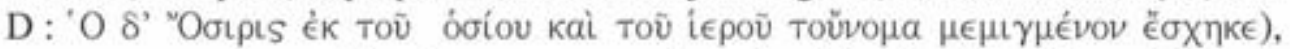

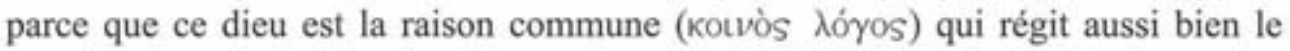

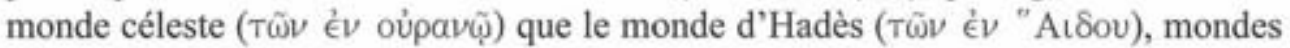
traditionnellement tenus, le premier, pour le lieu des réalités saintes (Tà $\delta^{\prime}$ ö $\sigma \iota \alpha$ ) et, le second, pour celui des réalités sacrées (Tà $\mu$ èv íepá). Les Rouleaux d'Hermès nous informent en effet, nous apprend-il ainsi, que les mêmes puissances divines reçoivent d'un peuple à l'autre des dénominations différentes : ainsi, de même que la puissance qui règle les révolutions du soleil s'appelle Horos en Égypte et Apollon en Grèce, de même celle qui est préposée au pneuma unificateur de l'univers est nommée Osiris par les Grecs, mais Sarapis par les Égyptiens.

Ces Rouleaux d'Hermès constituent manifestement une partie de ce que nous nommons maintenant Corpus hermeticum, ainsi désigné parce que s'y trouvent réunis des traités centrés sur la figure d'Hermès Trismégiste assimilé à Thoth. Il s'agit donc d'une littérature gréco-égyptienne qui vise à enseigner une sagesse révélée, indispensable au salut de l'âme. André-Jean Festugière lui a consacré une étude magistrale en quatre volumes, sous le titre général de La révélation d'Hermès 
Trismégiste ${ }^{1}$. Bien sûr, il mentionne ce passage de l'ouvrage de Plutarque, mais simplement comme preuve de l'existence au premier siècle de notre ère d'écrits hermétiques (vol. 1, p. 78) ${ }^{2}$. Toutefois il ne prétend pas que Plutarque ait lu lesdits Rouleaux d'Hermès. De fait, Plutarque lui-même se garde d'invoquer une quelconque lecture personnelle : il se contente de signaler leur contenu par les mots

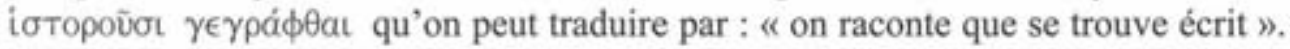
Il rapporte donc seulement une information de seconde main, voire de troisième main. Quoi qu'il en soit, il affirme avoir entendu parler de la gnose et du mysticisme hermétique. Est-ce assez pour suggérer, comme Jean $\mathrm{Hani}^{3}$, que, baignant dans un air du temps marqué par le goût des mystères, Plutarque se fait l'écho de l'hermétisme dans le finale de son traité sur le mythe d'Isis ? Yvonne Vernière", après s'être demandé si le mythe du dialogue Le visage qu'on voit apparaître dans l'orbe de la lune ne proviendrait pas d'une source hermétique, se rétracte presque en concluant que, touché par la vague montante de l'hermétisme, Plutarque, dont le syncrétisme reste toujours modéré, n'y sombre pas. Or Christian Froidefond ${ }^{5}$ écrit que dans Isis et Osiris Plutarque « frôle et côtoie » continuellement la Gnose et son syncrétisme. Plus récemment, John Dillon ${ }^{6}$ juge possible une influence, mais indirecte à partir d'une source commune pour des raisons de chronologie; en revanche, Abraham P. Bos ${ }^{7}$ estime inutile de supposer une quelconque influence sur Plutarque de la tradition hermétique, car elle est elle-même tributaire de la culture philosophique grecque et en particulier de l'aristotélisme.

Qu'en est-il exactement? Peut-on vraiment parler d'influence, qu'elle soit directe ou indirecte ? Et, dans le cas d'une réponse positive, ne s'agit-il que d'emprunts limités ou avons-nous affaire à une influence profonde ? Et, si l'influence n'a rien de superficiel, jusqu'où s'exerce-t-elle et comment s'explique-t-elle?

1

2

3

4

5

6

7

Parue à Paris en 1981.

C'est cette existence attestée par Plutarque qui me détermine à ne pas envisager ici la dette - également possible- de l'hermétisme envers Plutarque, bien que les textes conservés du Corpus hermeticum et sur lesquels s'appuie ma démonstration lui soient postérieurs (un paradoxe méthodologique qui se défend par le fait que ces textes reprennent des idées qui circulaient déjà vers la fin du $1^{\text {et }}$ siècle de notre ère, au moment de la rédaction du quatrième Êvangile).

La religion égyptienne dans la pensée de Plutarque, Paris, 1976, pp. 295-296.

Symboles et mythes dans la pensée de Plutarque, Paris, 1977, pp. 161, 336 et 340.

Plutarque. CEurres morales, t. $5 / 2^{\mathrm{c}}$ partie, CUF, Paris, 1988, p. 66.

"Plutarch and the Separate Intellect", in A. PÉrEZ JIMĖNEZ \& F. CAsAdesús BordoY (éds), Estudios sobre Plutarco: misticismo y religiones mistéricas en la obra de Plutarco, Actas del VII Simposio español sobre Phutarco, Palma de Mallorca, 2-4 sept. 2001, Madrid-Málaga, 2001, pp. $42-44$.

"The Distinction between 'Platonic' and 'Aristotelian' Dualism, illustrated from Plutarch's Myth in De facie in orbe lunae", op. cit. supra, pp. 66 et 68 (notes 53 et 54). 
Mon propos n'est pas de répondre à ces questions en procédant à une investigation exhaustive de l'œuvre de Plutarque, mais d'utiliser ce questionnement pour revisiter quelques passages significatifs des Moralia, en commençant précisément par le De Iside et Osiride.

Dans cet ouvrage je m'arrêterai plus spécialement sur trois passages.

Le premier (chapitres 2-3) se situe dans le préambule, où Plutarque affirme que c'est la connaissance qui transforme l'immortalité, de simple durée qu'elle est d'abord, en véritable vie et que c'est la connaissance qui fait le bonheur des dieux (351 D-E). Il ajoute que pour les mortels la connaissance se confond surtout avec l'aspiration à la vérité sur les dieux et que celle-ci ne saurait s'atteindre sans qu'on procède à une recherche philosophique $(352 \mathrm{C})$. Mais cette quête rationnelle est comparée au geste du myste qui dans le cadre d'une initiation aux Mystères est invité à

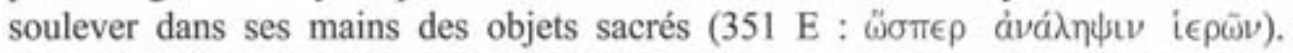
Autrement dit, dans la forme la plus élevée de son activité, la raison, loin d'exclure l'intuition, peut conduire à un savoir immédiat par révélation.

Or c'est précisément à un tel cheminement que nous convie l'ensemble de l'exégèse du mythe d'Isis et Osiris. En effet, la partie la plus philosophique de ce traité exégétique, celle où Plutarque expose ses idées personnelles (372 E) en conciliant théologie égyptienne et philosophie (371 A, cf. $378 \mathrm{~A}-\mathrm{B}$ ), se termine - voici notre deuxième passage (chapitres 77-78) - par l'affirmation de fulgurations de l'intellect capables de fournir une conception instantanée de l'intelligible le plus absolu. La spéculation de la philosophie se transmute alors en époptie, une forme de connaissance immédiate que Plutarque définit de la manière suivante. Je cite à travers la traduction de Christian Froidefond (382 D-E) : «... ceux qui, échappant grâce à la raison à ce domaine de l'opinion, des mixtes, du divers, s'élancent jusqu'au primordial, à l'indivisible, à l'immatériel et entrent en contact, totalement, avec la pure vérité qui lui appartient, ont l'impression de posséder, comme au terme d'une initiation, la philosophie dans son achèvement suprême. " Cependant cette vision illuminante réservée à ceux qui atteignent le plus haut degré de l'initiation aux mystères d'Éleusis n'égale pas en netteté celle que connaissent les âmes qui, délivrées de l'écran que constitue l'enveloppe charnelle, contemplent dans le monde invisible la beauté ineffable du divin $(383 \mathrm{~A})$. L'intellection à laquelle conduit la philosophie reste imparfaite : elle ressemble à un rêve flou (382 F). Une imperfection, nous assure le premier passage, qu'il est possible de compenser partiellement grâce à des pratiques cultuelles ascétiques, dont la finalité réside dans la connaissance de ce qui est premier, souverain et intelligible (352 A), laquelle n'est pas l'apanage des philosophes grecs. Des barbares peuvent donc accéder à une connaissance intime du divin et les Grecs ont parfois beaucoup à apprendre d'eux.

Le troisième passage (chapitre 80 ) à examiner n'est autre que le finale, consacré au kyphi, un développement annoncé (372 C-D) et qui n'a rien d'un appendice 
superfétatoire ${ }^{8}$. Plutarque s'y demande en effet pourquoi les prêtres égyptiens font brûler, le soir dans les temples, cette substance composée de seize ingrédients différents, et il explique que leur mélange se trouve en parfaite harmonie avec la qualité de l'air nocturne, composé de toutes les lumières émises par les astres, si bien que cette fumigation met le lieu sacré en adéquation totale avec l'ordre du cosmos, tout comme le font, le jour, les fumigations de résine et de myrrhe, et que les exhalaisons ainsi évoquées du parfun symbolisent l'amalgame parfaitement homogénéisé des vérités révélées au terme des interprétations accumulées tout au long de l'enquête sur le sens théologique du mythe. «Les Égyptiens », conclut Plutarque, « ont donc raison de faire brûler de jour la résine et la myrrhe, qui sont des substances simples et tirent leur origine du soleil, et à la tombée de la nuit l'ensemble des composants du kyphi, qui réunit en lui toutes sortes de propriétés différentes. " ${ }^{9}$

Voilà trois passages dont il est difficile de ne pas rapprocher le mysticisme de celui de certains traités du Corpus hermeticum. Certes, Platon ${ }^{10}$ soutient que le frottement, les unes contre les autres, des définitions, des perceptions et des opinions fait jaillir dans les objets de la réflexion la lumière de l'intellect. Mais il s'agit d'une métaphore pour désigner les éclaircissements apportés par l'intelligence. Naturellement les cérémonies initiatiques d'Éleusis comportaient un brusque passage de l'obscurité à une clarté éblouissante avant l'ostension des symboles sacrés. Toutefois nous n'avons affaire qu'à un rite, alors que, chez Plutarque, nous sommes en présence d'un acte cognitif, dont le médium est précisément la lumière, qui, selon le traité intitulé Poimandrès (chapitre 21), constitue la substance de l'homme comme celle de Dieu son père : "parce que », répondis-je", " c'est de lumière et de vie qu'est constitué le Père de toutes choses, de qui naquit l'Homme. - Tu dis bien : lumière et vie, voilà ce qu'est le Dieu et Père, de qui est né l'Homme. Si donc tu apprends à te connaître comme étant fait de vie et de lumière et que ce sont là les éléments qui te constituent, tu retourneras à la vie. ${ }^{12}$ La gnose hermétique affirme donc qu'il existe une consubstantialité lumineuse entre l'homme et le divin et que c'est cette consubstantialité qui rend possible la connaissance, ainsi que le confirme cette autre déclaration: «... ce qui en toi regarde et entend, c'est le Verbe du Seigneur, et ton Noûs est le Dieu Père : ils ne sont pas séparés l'un de l'autre, car

8 Voir mon article "Un parfum d'Égypte : le kuphi et son pouvoir imaginaire", in J. THомAs, P. Carmignani, J.-Y. Laurichesse (éds), Saveurs, senteurs : le goût de la Méditerranée, Perpignan, 1998, pp. 59-71.

9 Page 384 B-C. Traduction de C. FroIDEFond.

10

Voir la Lettre 7, 344 b.

11 Le traité rapporte un dialogue entre Hermès Trismégiste lui-même et Poimandrès, nom donné à I'intellect suprême, principe des principes, en d'autres termes Dieu Père (voir chapitre 6).

12

Traduction de A.-J. Festugiére (Hermès Trismégiste, t. 1, CUF, Paris, 1999, $1^{\text {ère }}$ éd. 1946). 
c'est leur union qui est la vie. ${ }^{13}$ Or c'est justement ce que présupposent et l'époptie à laquelle conduit la spéculation philosophique recommandée par Plutarque et l'extase religieuse qui la prolonge.

Quant aux exhalaisons du kyphi dont l'expansion à travers l'air de la nuit unit, comme par osmose, l'espace sacré du temple à celui du ciel, si elles renvoient à la théologie astrale de Platon ${ }^{14}$ de même qu'à la théorie stoĩcienne de la sympathie universelle que n'ignore pas Plutarque, elles relèvent surtout de la croyance en la présence secrète de relations d'antipathie ${ }^{15}$ ou de sympathie entre les réalités du monde, des relations dont on peut se servir en médecine ou en magie, quand on connait les propriétés élémentaires de la matière, notamment des minéraux et des végétaux ; or cette doctrine, développée par Bolos de Mendès traditionnellement tenu pour le fondateur de l'alchimie ${ }^{16}$ et dont le traité Livre des Sympathies faisait encore autorité à l'époque de Plutarque dans le domaine des sciences de la nature ${ }^{17}$, se retrouve en particulier dans l'affirmation que le monde est traversé d'énergies de toute sorte ${ }^{18}$.

Il apparaît de plus en plus difficile d'écarter l'hypothèse d'une utilisation étendue de l'hermétisme par Plutarque, ce qui nous ramène aux Rouleaux dits d'Hermès mentionnés au chapitre 61 du De Iside et Osiride. Il est clair que notre auteur n'en exploite pas les informations que sur des questions d'étymologie ${ }^{19}$. Du coup, une connaissance relativement massive de ces textes ne saurait se concevoir sans une lecture personnelle. Pourquoi, dans ces conditions, se retranche-t-il derrière l'impersonnel i $\sigma т о р о \overline{\sigma \iota ~(375 ~ F), ~ a l o r s ~ q u ' i l ~ l a i s s e ~ e n t e n d r e ~ n e t t e m e n t ~ q u ' i l ~ a ~ l u ~ l e s ~ a u t r e s ~ d o c u-~}$ ments auxquels il renvoie, tels les hymnes sacrés d'Osiris (chapitre 52, 372 B) qu'il cite directement ou les Écrits phrygiens (chapitre 29,362 B) dont il récuse le contenu ? La réponse dépend peut-être de l'examen du reste de l'œuvre de Plutarque. Trois autres ouvrages des Moralia présentent en effet des passages qui invitent fortement, sinon à conclure à des traces d'emprunts à l'hermétisme, du moins à opérer

13

14

15

16

17

18

19

Chapitre 6, traduction d'A.-J. FESTUGIERE.

Voir le Timée, $41 \mathrm{~d}-42$ e, où, après avoir créé les dieux visibles que sont les astres, le démiurge y sème toutes les âmes qu'il a formées.

Cf. Quaest. conv. 2, 7, 1, 641 B-C.

Pour un point de vue plus nuancé, voir Jackson P. HershBELL. ("Democritus and the Beginning of Greek Alchemy ", Ambix, 34/1, march 1987, 5-20), qui estime que le véritable fondateur de l'alchimie grecque n'est autre que Démocrite d'Abdère, dont précisément Bolos de Mendès a pu se réclamer.

Voir A.-J. FestugtéRE, op. cit. supra, t. 1, p. VII.

Voir Hermès Trismégiste, traité X, chapitre 22 (CUF, t. 1, 1999).

On pourrait aussi, par exemple, noter qu'une des explications avancée par Plutarque sur le culte égyptien des animaux (chapitre 72,380 B), à savoir que la divinisation des animaux est une invention humaine à des fins politiques qui varie d'une ville à l'autre, provient sans aucun doute du traité intitulé Asclépios (chapitre 37, CUF, t. 2, 1992, p. 348). 
des rapprochements avec ce dernier. Ces rapprochements touchent à quatre champs d'interrogation : l'anthropologie, la gnoséologie, la psychologie et la physique.

Commençons par la nature de l'homme. Dans son opuscule Si la maxime Vis caché est une bonne maxime, Plutarque s'attaque à l'épicurisme en faisant valoir qu'il s'agit là d'un précepte contre nature, car l'être humain est naturellement appelé à s'épanouir dans la pleine lumière du jour, sans laquelle deux aspirations profondes et innées ne peuvent que rester frustrées, le besoin d'être connu et celui de connaître. Pour expliquer cette double aspiration, il rappelle que les Anciens donnaient aux hommes le nom de photes et il suggère que c'est à cause d'une étroite parenté avec la lumière $(p h o s)^{20}$. Or la conviction que l'espèce humaine possède un intellect de substance lumineuse, un don divin, court partout dans le Corpus hermeticum, tout particulièrement dans le traité intitulé Poimandrès (chapitre 6), mais également dans les Mémoires authentiques de Zosime de Panopolis, qui reprend la même paronomase ó $\phi \omega ́ s$, Tò $\phi \tilde{s}$ pour assimiler à la lumière la partie spirituelle de l'homme ${ }^{21}$. Bien sûr, avec cet alchimiste de Haute-Égypte nous avons affaire à un personnage des troisième et quatrième siècles, mais, observe Michèle Mertens $\mathrm{s}^{22}$, les théories gnostiques nourrissent les spéculations de l'alchimie. Le problème vient de ce que Plutarque renvoie, cette fois sans les nommer, à certains philosophes pour qui l'âme est consubstantielle à la lumière solaire, parce qu'elle est spontanément attirée par tout ce qui est lumineux et qu'elle supporte mal l'obscurité (1130 B). Or les Pythagoriciens, aux dires de Plutarque lui-même ${ }^{23}$, comparent le corps à une lampe et l'âme à sa lumière éclairante. Et, à l'époque classique, ils ne sont pas les seuls à le faire. Héraclide du Pont, un astronome rangé par Diogène Laërce $(5,86)$ au nombre des disciples d'Aristote bien qu'il ait été l'auditeur des Pythagoriciens et de Speusippe, enseigne que l'âme est faite d'éther et que sa substance provient de la Voie Lactée ${ }^{24}$. On peut done en conclure que la psychologie gnostique plonge une partie de ses racines dans la philosophie pythagorico-platonicienne ${ }^{25}$, et il est, à peu près certain, que cette conception de l'âme ne vient pas à l'idée de Plutarque par le détour de la gnose hermétique. Toutefois rien n'interdit qu'il ait pu dans son esprit associer les gnostiques à ces philosophes évoqués anonymement.

20

21

22

23

24

25

Voir chapitre 6, 1130 A-B.

Mémoires authentiques, 1, 10 (Mertens M., Les Alchimistes grecs, t. $4 / 1^{\mathrm{e} r e}$ partie, CUF, Paris, 1995).

Op. cit. supra, p. 96 , notes 64 et 65 .

Voir Quaest. Rom., 72, 281 B.

Voir le frag. 98 a, b, c et d, ainsi que les fragments 99 et 97 (WHERLL). Dans son commentaire Fritz WherLu (Die Schule des Aristoteles. Herakleidos Pontikos, Basel/Stuttgart, 1969, p. 93) fait remarquer que la pensée d'Héraclide découle de celle de Platon qui assimile les âmes aux étoiles (Politeia, 10, 621 b).

Voir A.-J. FestuGièRE, op. cit. supra, t. 1, p. 282. 
Le Dialogue Le démon de Socrate renforce cette impression. Nous intéresse tout particulièrement la longue intervention de Simmias, que l'on peut regarder comme l'un des porte-parole de l'auteur. Il interprète les allusions que faisait Socrate à son démon comme la mention d'une expérience de communication extraordinaire avec un être céleste, qui, sans le truchement de la voix et du langage articulé, entrait intellectuellement en contact avec le philosophe, dont l'esprit pur de toute passion rendait possible un tel commerce ${ }^{26}$. Certes, de nouveau nous rencontrons une référence à Platon avec une comparaison de l'âme à une marionnette mue par des impulsions $^{27}$. Cependant, nous ne trouvons pas trace, chez Platon, d'une communication purement intellectuelle entre deux lumières qui s'effleurent, celle d'un démon, c'est-à-dire d'un esprit désincarné, et celle de la partie supérieure d'un mortel encore incarné, à savoir son noûs. Voici ce que Plutarque écrit : «Car, en réalité, la manière dont nous nous communiquons nos pensées par le moyen du langage parlé ressemble à un tâtonnement dans les ténèbres, tandis que les pensées des démons, qui sont lumineuses, brillent dans l'âme des hommes démoniques ; elles n'ont pas besoin des paroles et des mots que les hommes emploient comme signes pour communiquer entre eux, ce qui fait qu'ils n'ont que des figures et des images de leurs pensées, dont ils ignorent la réalité même, hormis les hommes qui, comme je l'ai dit, reçoivent une lumière spéciale qui leur vient des démons. ${ }^{28}$ Or Poimandrès révèle à Hermès Trismégiste que l'Homme, plus précisément l'homme archétypal, « de vie et de lumière qu'il était, se changea en âme et en intellect, la vie se changeant en âme, la lumière en intellect ${ }^{29}$ Et cette lumière dont est fait l'intellect n'est autre, comme on l'a vu précédemment, que celle de Dieu, Père de cet homme archétypa $^{30}$. Donc te connaître, dit encore Poimandrès à Hermès, c'est savoir que « ce qui en toi regarde et entend, c'est le Verbe du Seigneur et que ton Noûs est le Dieu Père .... ${ }^{31}$ L'union du logos, autrement dit de la rationalité pure, - qu'il importe de distinguer de la parole articulée-, et de la lumière de l'intelligence constitue ainsi le fondement de la connaissance mystique, caractérisée par des visions, sans qu'il y ait la moindre démonstration. Le Poimandrès commence par une vision et se poursuit par son explication, mais le préambule souligne que tout s'effectue en pensée, y compris la communication ${ }^{32}$. Comment dès lors l'envisager autrement que sur un mode lumineux du genre de celui qu'évoque le discours prêté au pythagoricien

Voir le chapitre 20, passim.

Chapitre 20, 588 F, cf. Lois 1, 644 d-e.

Chapitre 20, 588 B-C. Traduction de J. HANI (Plutarque. Euvres Morales, t. 8, CUF, Paris, 1980).

Hermès Trismégiste, traité 1, 17. Traduction d'A.-J. Festugiére (t. 1, CUF, 1999).

Voir op. cit. supra, chapitre 21.

Op. cit. supra, chapitre 6. Traduction d'A.-J. FestugièrE.

Op. cit. supra, chapitre 1 , cf. Hermès Trismégiste, traité 5. 
Simmias ? Plutarque développerait-il la théorie de Pythagore au moyen d'un aspect de la connaissance gnostique?

Autre passage remarquable du Démon de Socrate : la réécriture du mythe d'Er que constitue le récit de Timarque rapporté toujours par Simmias (chapitres 21-22) décrit la structure du monde au moyen de quatre principes, la Vie (Zwí), le Mouvement, la Génération et la Corruption, les deux premiers étant reliés par l'Unicité (Movás), le couple des premiers étant lié au troisième de ces quatre principes par l'Intellect (Noũs), et l'ensemble des trois premiers au quatrième par la Nature naturante (申úøıs) ${ }^{33}$. Dans ce système où l'on peut déceler aussi un remaniement des cinq genres suprêmes que sont pour Platon ${ }^{34}$ l'être, le mouvement, le repos, le même et l'autre, il est frappant de constater que le sommet de cette hiérarchisation est occupé par la Vie. Or nous venons de voir ce qu'il en est des catégories-clés de la Vie et de l'Intellect dans le Poimandrès. Par ailleurs, le discours sacré d'Isis dans le traité Pupille du monde ${ }^{35}$ raconte comment Dieu Premier Père créa la nature naturante qu'est Physis (sections 10-11). D'autre part, le traité 13 (D'Hermès Trismégiste à son fils Tat: discours secret sur la montagne, concernant la régénération et la règle du silence) célèbre l'Un en affirmant que l'unification de le Vie et de la Lumière entraîne

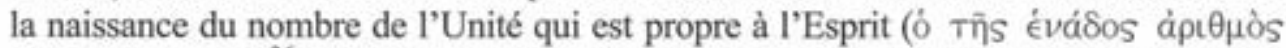
Toũ $\pi \nu \in \cup \dot{\mu} \mu \mathrm{T} O S)^{36}$. Nous avons donc affaire aux trois liens grâce auxquels Plutarque assure la cohésion du cosmos, et le principe de vie qui occupe le sommet de sa hiérarchisation se confond avec Dieu dans la révélation du Poimandrès ${ }^{37}$. Plutarque a-t-il remanié le matériel platonicien à la lumière du mysticisme gnostique ?

On peut de nouveau se poser la question en lisant, dans le dialogue Les délais de la justice divine, le mythe de Thespésios, une autre réécriture du mythe d'Er. Et ce, pour au moins un passage, celui de l'évocation de la purification des âmes avant leur recyclage ${ }^{38}$. L'enfer ouranien où nous sommes entrainés comprend trois étangs remplis l'un d'or en ébullition, l'autre de plomb très froid, et le troisième de fer également liquide, mais tiède. Trempées successivement plusieurs fois dans ces trois lacs, changeant de couleur, de consistance et de forme, les âmes reprennent leur éclat originel uniformément lumineu ${ }^{39}$. Comment ne pas se dire que Plutarque

Chapitre 22, $591 \mathrm{~B}$.

Voir en particulier Le Sophiste, 254 b.

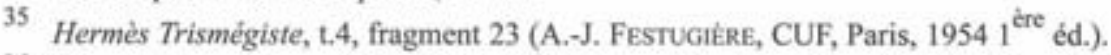

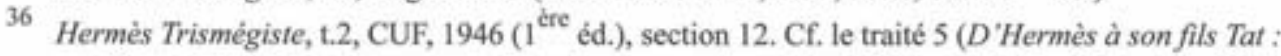
que Dieu est à la fois inapparent et le plus apparent), section 2 (Hermès Trismégiste, t. 1, CUF, $1946,1^{\text {ere }}$ éd.), où Dieu est dit être la source de l'Un, bien loin de se confondre avec l'Un.

Hermès Trismégiste, t. 1, traité 1, section 9.

39

Chapitre 30, 567 C-D.

Chapitre 26, $565 \mathrm{C}$. 
pense les châtiments infernaux sur le modèle des transformations de la matière opérées par les métallurgistes ou les teinturiers et mises en recettes par ceux qu'on appelle les alchimistes ${ }^{40}$ ? Les procédés de la fusion et de la trempe utilisés métaphoriquement pour la régénération de la substance immatérielle de l'âme renvoient fortement aux spéculations de l'alchimie sur la transmutation des métaux ${ }^{41}$. Il n'est pas non plus impossible que cette image, absente de l'enfer platonicien ${ }^{42}$, soit inspirée par les écrits gnostiques qui décrivent la création de l'âme du monde comme un mélange de type alchimique, fait de souffle, de feu, de substances inconnues, et à l'aide également d'incantations secrètes ${ }^{43}$.

Pour conclure provisoirement sur ce dossier très embryonnaire, je voudrais, outre le constat que les rappochements examinés offrent plus de ressemblance avec l'hermétisme qu'avec d'autres doctrines, esquisser quelques réflexions qui pourraient ouvrir tout un programme d'investigations complémentaires ${ }^{44}$.

D'abord, entre la pensée de Plutarque et l'hermétisme gnostique les points de convergences ne se limitent pas à ceux des seuls traités passés en revue. L'un des plus significatifs est un imaginaire de type solaire qui des deux côtés accorde au soleil un rôle décisif dans la structure du monde : image du Dieu unique Apollon dans $L^{\prime} E$ de Delphes ${ }^{45}$, siège de l'Intellect dans le Démon de Socrate ${ }^{46}$ et source du cycle de la vie dans Le visage qu'on voit apparaitre dans l'orbe de la lune ${ }^{47}$, démiurge du Dieu Père dans le traité 16 (D'Asclépios au roi Ammon : définitions) du Corpus hermeticum ${ }^{48}$, de même que lien du ciel et de la terre ${ }^{49}$ et opérateur d'une création continuée sans fin ${ }^{50}$.

Il est ensuite remarquable que Plutarque cultive un goût prononcé pour la figure de pensée que constitue la révélation, dont André-Jean Festugière souligne qu'el-

Voir le Papyrus de Leyde (R. Hall.eux, Les Alchimistes grecs, t. 1, CUF, Paris, 1981), passim.

Voir J. BoulogNe, « L'enfer ouranien de Plutarque », in J. Thomas (éd.), L'imaginaire religieux gréco-romain, Perpignan, 1994, 217-234. consacrées à la religion de Plutarque et qu'a réunies Rainer HIRSCH-LuIPOLD sous le titre Gott und die Götter bei Plutarch (Berlin, New York, 2005) : la question de l'hermétisme n'est abordée nulle part.

Chapitre 21, 393 C-D.

Chapitre 22, $591 \mathrm{~B}$.

Chapitre 30, 945 C, ef. chapitre 28, 943 A.

Hermès Trismégiste, t. 2, CUF, sections 5 et 18.

Ibidem, section 5.

Ibidem, sections 18-19.
} 
le est caractéristique de l'illumination gnostique ${ }^{51}$. De fait, chacun des traités du Corpus hermeticum délivre sans démonstration et sur le mode mythique un enseignement d'origine divine transmis soit à Hermès, soit par Hermès, sur des vérités premières ou dernières. Or, pour exposer ses conceptions eschatologiques et sotériologiques, Plutarque ne procède pas autrement. Nous l'avons relevé avec les mythes de Tìmarque et de Thespésios. On pourrait ajouter celui de Sylla dans le dialogue Le visage qu'on voit apparaitre dans l'orbe de la lune. Ce Sylla est un Carthaginois qui prétend tenir d'un voyageur anonyme des vérités révélées sur et par Cronos lui-même, et peut-être aussi par un prétendu parchemin sacré, redécouvert à Carthage après de longues années passées dans la terre ${ }^{52}$, une variante de la figure de pensée qu'est le brusque dévoilement de mystères. Un autre dialogue, $\mathrm{La}$ disparition des oracles, nous fournit un exemple supplémentaire d'un tel type de rhétorique : Cléombrote, un Spartiate grand voyageur, lui également en quête de savoir théologique et qualifié sans doute pour cette raison de "saint homme",53 , rapporte les réponses d'un barbare de la côte de la Mer Érythrée à ses questions sur la divination, les démons, le nombre des mondes et le temps, toutes réponses énoncées mythiquement ( $\mu \cup \theta 0 \lambda$ 이votos), mais présentées comme le résultat d'un face-à-

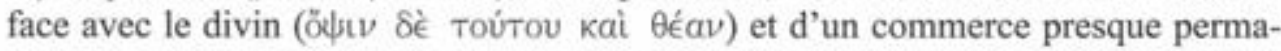
nent avec les démons, si bien qu'elles deviennent l'équivalent d'une initiation à des

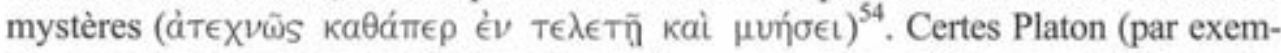
ple, avec le personnage de Diotime dans le Banquet ) utilise déjà le procédé. Mais rien ne nous ordonne d'exclure de l'esprit de Plutarque le modèle de la pratique hermétique, dont la somme interprétative Isis et Osiris nous apprend qu'il la connaît bien.

Ici, nous arrivons à une troisième série de remarques qui touchent à l'éclectisme dogmatique de notre auteur, différent du dogmatisme éclectique de Cicéron. Intimement convaincu de l'incapacité de l'esprit humain à s'emparer totalement de la vérité, notamment dans le domaine des dieux, Plutarque pense que cette dernière, par son caractère universel, transcende les idiotismes individuels et les particularismes ethniques. Elle n'est donc ni barbare, ni grecque ${ }^{55}$. Nul discours ne saurait non plus en revendiquer le monopole, d'autant moins qu'aucun ne parvient à éviter une part d'erreur, si bien que, pris séparément, les discours ont tous tort, alors que réunis dans une même somme, ils ont tous ensemble raison ${ }^{36}$. Cette épistémologie

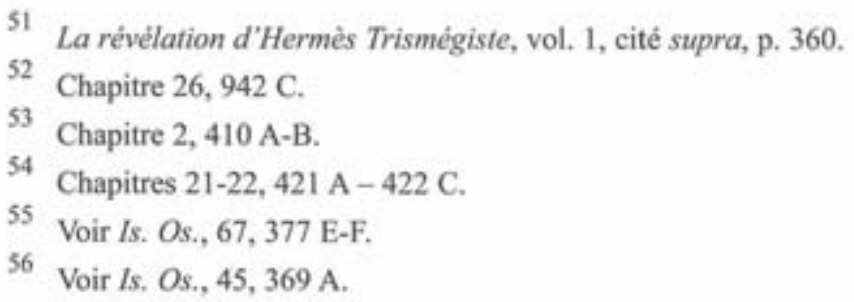


assimile la quête du vrai au tri du chercheur d'or qui, dans son tamis, sépare les pépites du reste afin de rassembler des paillettes dispersées, et en conséquence elle invite à prendre en considération tous les discours sans exception, ceux des poètes, des législateurs et des philosophes ${ }^{57}$, tout comme les mythes ${ }^{58}$ et la tradition populaire de la foi ancestrale $^{59}$. Du coup, la fidélité exclusive à une doctrine unique perd toute signification. Reprocher, entre autres, à Plutarque son hétérodoxie en matière de platonisme, par exemple sur la nature primordiale d'Éros dont Platon fait un simple démon ${ }^{60}$, ne présente guère de pertinence. De plus, une telle épistémologie oblige, selon Plutarque, à adopter un point de vue à la fois anthropologique et historique. C'est ainsi qu'il estime que l'humanité a progressé en théologie, lorsque, pour éviter de rendre la Providence responsable du mal, elle a inventé, grâce à Zoroastre ou à Orphée, une catégorie d'êtres intermédiaires entre les hommes et les dieux ${ }^{61}$, et qu'il retrace l'histoire de cette doctrine depuis son origine anonyme jusqu'aux philosophes grecs, Héraclite, Empédocle, Pythagore, Anaxagore, Aristote, Platon (pour suivre l'ordre de l'énumération du texte), en passant par les Mages perses et les Chaldéens ${ }^{62}$. Dans ces conditions, l'ouverture de Plutarque à des conceptions ou à des théories étrangères, mais compatibles et convergentes avec les principes qui structurent sa vision du monde, ne peut qu'être très grande, surtout quand la rencontre s'effectue avec un système de représentations lui-même très éclectique, comme c'est le cas avec l'hermétisme ${ }^{63}$.

Autant d'attendus qui ajoutent au constat et plaident de façon plausible en faveur de la thèse d'une contribution de l'hermétisme à la réflexion de Plutarque et à son enrichissement d'une manière aussi bien ponctuelle que diffuse. Sans doute n'est-il pas pour rien dans l'importance que prend chez lui le paradigme de la crase ${ }^{64}$, particulièrement actif dans les spéculations des alchimistes. C'est malgré tout trop peu pour parler d'influence. Il vaut mieux se dire qu' aux yeux de Plutarque l'hermétisme contient des parcelles de vérité dont il serait dommageable de ne pas tenir compte, et pour cette raison il semble vraisemblable que la curiosité intellectuelle a dû le pousser à lire tel ou tel des traités de cette littérature, mais pas les écrits de magie et de sciences occultes dont André-Jean Festugière dit qu'ils forment l'hermétisme

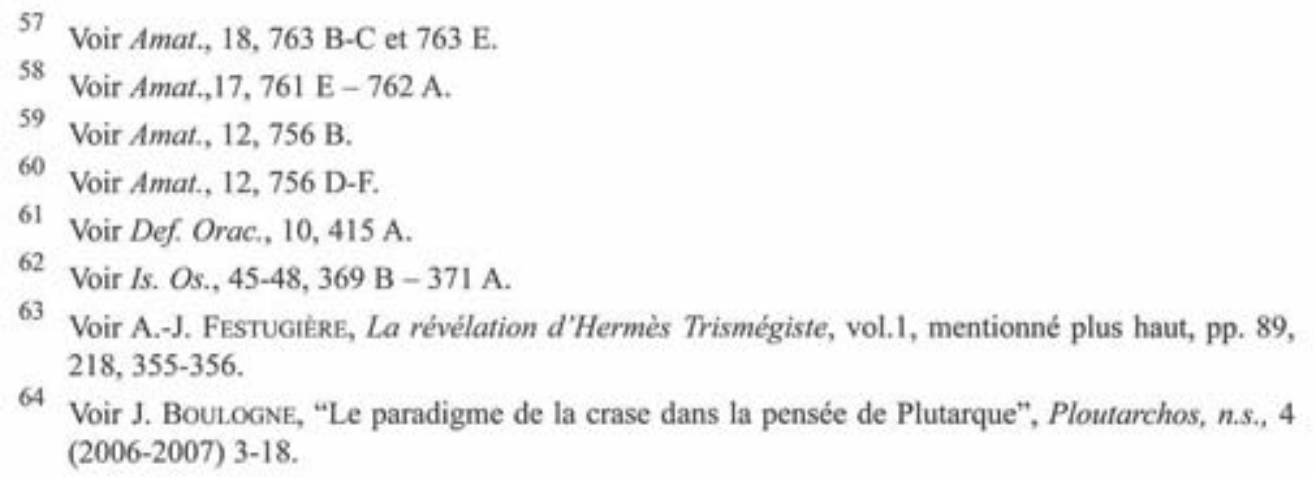


populaire ${ }^{65}$. Dès lors, les Rouleaux dits d'Hermès, mentionnés dans l'ouvrage Isis et Osiris comme une source d'information indirecte, renverraient, non pas à la totalité du Corpus hermeticum existant de son temps, mais uniquement à ceux de ses écrits qui relèveraient de cet hermétisme populaire, jugés par lui d'un intérêt trop limité pour mériter une lecture, à la différence des écrits de l'hermétisme non populaire ou plus philosophique qu'il a pu, eux, éprouver le besoin de lire.

\section{BIBLIOGRAPHE}

FESTUGIÈRE A.-J.,

- La révélation d'Hermès Trismégiste, Paris, 1981 (vol. L'astrologie et les sciences occultes; vol. 2 Le dieu cosmique ; vol. 3 Les doctrines de l'äme ; vol. 4 Le dieu inconnu et la gnose).

- Hermès Trismégiste, t. 1, CUF, Paris, 1999 (1 ${ }^{\mathrm{e} r e}$ éd. 1946).

- Hermès Trismégiste, t. 2, CUF, Paris, 1992 (1 $1^{\text {tre }}$ éd. 1946).

- Hermès Trismégiste, CUF, t. 4, Paris, 2002 ( $1^{\text {ère }}$ éd. 1954). 
(Página deixada propositadamente em branco) 


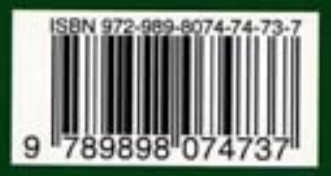

